\title{
Estudo fatorial exploratório das dimensões comportamentais de adesão em pessoas com diabetes mellitus tipo 1 e tipo 2 - Dimensões comportamentais de adesão'
}

\author{
Exploratory factor study of adherence behavioral dimensions in diabetes mellitus patients type 1 and type \\ 2 - Adherence behavioral dimensions
}

Artigo Original | Original Article

Ilda Massano Cardoso, PhD (1, 2a), Fernanda Daniel, PhD (1b), Vítor Rodrigues, PhD (2c), Manuela Carvalheiro, PhD (2d)

(1) Instituto Superior Miguel Torga, Coimbra, Portugal.

(2) Faculdade de Medicina da Universidade de Coimbra, Coimbra, Portugal.

(2) CEISUC da Faculdade de Economia da Universidade de Coimbra, Coimbra, Portugal.

(a) Revisão da literatura, conceção da investigação, interpretação e discussão dos resultados e redação do manuscrito.

(b) Revisão crítica no âmbito científico da estatística. Contributo na redação do manuscrito.

(c) Conceção e desenho de investigação. Revisão da redação final do manuscrito.

(d) Revisão crítica no âmbito científico da diabetes. Revisão da redação final do manuscrito.

Autor para correspondência | Corresponding author: Ilda Massano Cardoso Largo da Cruz de Celas, n¹, 3000-132 Coimbra, Portugal; e-mail: ildamassano@ismt.pt

\section{Palavras-Chave}

Diabetes mellitus

Dimensões comportamentais

Análise fatorial

Adesão

\section{Keywords}

Diabetes mellitus

Factorial analyses

Behavioral dimensions

Adherence

\section{RESUMO}

Objetivos: Analisar as dimensões comportamentais associadas à adesão em pessoas com diabetes mellitus (DM). Testar o modelo teórico composto por fatores internos e relacionais ao paciente tendo por base oito instrumentos de autorresposta.

Métodos: 347 doentes a frequentar consultas de diabetes do Serviço de Endocrinologia, Diabetes e Metabolismo no Centro Hospitalar e Universitário de Coimbra e na Associação Portuguesa de Diabéticos de Portugal que aceitaram colaborar no preenchimento de instrumentos de autorresposta. Os instrumentos utilizados foram a Escala de Autoestima de Ronsenberg, WHOQOL-Bref, Escala de Satisfação com o Suporte Social, Medida de Adesão aos Tratamentos, Attitudes Towards Doctors and Medicine, Inventário de Depressão de Beck, O Perfil de Saúde da Diabetes e Questionário de Autocuidados da Diabetes. Foi realizada uma análise fatorial exploratória (AFE) com recurso ao método das componentes principais. A adequação dos dados à $A F E$ foi verificada por meio dos testes de Kaiser-Meyer-Olkin (KMO) e esfericidade de Bartlett.

Resultados: A estrutura fatorial encontrada no caso da DM tipo 1 é de seis componentes com uma variância total explicada de $70,8 \%$, na DM tipo 2 com tratamento insulínico é de sete componentes com uma variância total explicada de 74,9\% e, no caso da DM tipo 2 com tratamento oral, o número de componentes é de seis com uma variância total explicada de $73,4 \%$.

Conclusões: As saturações mais elevadas corresponderam aos construtos a que teoricamente deveriam pertencer. Na DM tipo 1, o fator terapêutico desapareceu, associando-se ao autocuidado. Na DM tipo 2 oral, esta componente emergiu como uma dimensão. Da análise forçada a dois fatores verificou-se que o suporte social não saturou de forma adequada no fator relacional, mas sim no fator interno. Conclui-se a satisfação social pode ser percebida como uma dimensão interna de adesão.

\section{ABSTRACT}

Aim: The current study sought out to conduct an exploratory factor analysis in order to examine behavioral dimensions associated with adherence in diabetes mellitus (DM) patients. Additionally, our goal was to test a theoretical model consisting of internal and relational factors.

Methods: Three hundred and forty seven patients attending consultations at the Serviço de Endocrinologia, Diabetes e Metabolismo of the Centro Hospitalar Universitário de Coimbra and at the Associação Portuguesa de Diabéticos de Portugal. The self-report instruments were Rosenberg Self-esteem Scale, WHOQOL-Bref, Escala de Satisfação com o Suporte Social, Medida de Adesão aos Tratamentos, Attitudes Towards Doctors and Medicine, Beck Depression Inventory, Diabetes Health Profile e Questionário de Autocuidados da Diabetes. Exploratory factor analysis was used to assess the instrument's dimensionality with Kaiser-Meyer-Olkin and Bartlett's Sphericity Test.

Results: For type $1 \mathrm{DM}$ the factor structure found included six components with a total explained variance of $70.8 \%$. For type $2 \mathrm{DM}$ (insulin treatment) seven components were found explaining a total variance of $74.9 \%$. For type 2 diabetes (oral treatment) six factors accounting for $73.4 \%$ of total explained variance were found.

Conclusions: The highest component loadings were found in constructs that should theoretically belong to the corresponding factors. In the case of type $1 \mathrm{DM}$ the therapeutic factor disappears, combining the self-care factor, and in the case of type $2 \mathrm{DM}$ (oral), this component emerged as a dimension. A forced two-dimentional factor analysis indicated that social support load in internal factor. These findings led us to reflect on the suitability of the instrument for the assessment of social support, instead of the model itself. An explanation based on the multidimensionality of therapeutic adherence was achieved considering that internal structure and semantics conditioned our results. 


\section{INTRODUÇÃO}

A análise dos determinantes ou fatores de adesão tem sido o tema de diversos trabalhos no âmbito do estudo das doenças crónicas. A adesão ao tratamento revela uma natureza multifatorial, sendo influenciada por fontes diversas e estas podem constituir-se, de acordo com Leventhal e Cameron (1987), em grupos ou componentes diferentes. Sackett e Haynes (1976) identificaram mais de duas centenas de variáveis que parecem estar relacionadas com a adesão ao tratamento. Estas variáveis são agrupadas, segundo estes autores, em cinco categorias: as características do doente, as características do tratamento, as características da doença, o relacionamento com os profissionais de saúde e as variáveis organizacionais e estruturais. Com efeito, Sanchez-Sosa (2001) propõe uma classificação mais reduzida, operacionalizada em quatro componentes: o doente, a equipa de saúde, a doença em si e os fatores associados às condições do macrossistema (traços socioeconómicos, geográficos e culturais de um determinado país). É reduzida a probabilidade de que estes quatro fatores "atuem" de forma isolada, sendo de esperar que os fatores se entrecruzem e interajam, produzindo vários graus de adesão ao tratamento.

Entre os determinantes que têm vindo a ser identificados salientamos, no presente estudo, a importância das características comportamentais internas e relacionais no caso da DM, muito embora a literatura reporte a relevância de variáveis externas ao indivíduo.

Sabe-se que as variáveis demográficas predizem fracamente a adesão aos autocuidados gerais na diabetes. Tal não acontece com a idade, que apresenta impacto na adesão (Warren e Hixenbaugh,1998). Importa, contudo, referir que a relação entre a idade e escolaridade surge fracamente associada à adesão ao regime alimentar (Brooks, 2002). Ainda nos determinantes demográficos face à adesão, estudos revelam que adolescentes com DM tipo 1 aderem menos ao tratamento do que as crianças, nomeadamente em relação à regularidade da toma de insulina, exercício físico, cuidados alimentares e automonitorização da glicemia capilar (Hirschberg, 2001).

Os fatores relacionais, tal como o apoio social apresentam efeitos combinados com a idade (Warren e Hixenbaugh, 1998). Estes autores verificaram a existência de uma menor probabilidade de aderir à terapêutica quando estão em contexto de trabalho, em idades compreendidas entre os 18 e os 50 anos. A probabilidade de não adesão aumenta nos doentes com idade superior a 50 anos quando em presença de amigos. Este facto ilustra bem o impacto das relações sociais no desempenho terapêutico dos doentes com diabetes. No que respeita aos efeitos combinados do sexo com o apoio social, Warren e Hixenbaugh (1998) afirmam tratar-se de um fator preponderante na adesão, constatando que os homens apresentam uma menor adesão ao tratamento na presença de colegas de trabalho do que as mulheres.

Numa revisão do estudo publicado por Ryan (1997) foram encontradas diferenças significativas quanto à relação idade do doente e às crenças na saúde, podendo estas ser determinantes da adesão. A perceção da gravidade, pelos indivíduos mais novos, parece promover a adesão à terapêutica enquanto para os mais velhos são os benefícios percebidos da adesão ao tratamento que os fazem ter mais autocuidados na diabetes.

Tal como descrito anteriormente, são múltiplos os fatores que poderem determinar a adesão. Assim, através de um modelo meramente exploratório neste trabalho procurou explorar-se as variáveis que podem associar-se à adesão.

\section{MÉTODO}

$\mathrm{Na}$ avaliação e refinamento dos instrumentos de avaliação é frequente recorrer-se a procedimentos estatísticos, nomeadamente à análise fatorial exploratória (AFE). Este conjunto de técnicas tem como principal objetivo encontrar uma estrutura subjacente numa matriz e determinar o número e a natureza das variáveis latentes (fatores) que melhor podem representar um conjunto de variáveis observadas (Brown, 2006).

De acordo com Pasquali (2003) a análise fatorial (AF) assenta num pressuposto em que as variáveis empíricas ou observáveis (normalmente traduzidas por indicadores) podem ser explicadas por um número menor de variáveis hipotéticas, comumente designada por fatores.

\section{Participantes}

O presente estudo foi conduzido numa amostra de 347 indivíduos a frequentar consultas de diabetes do Serviço de Endocrinologia, Diabetes e Metabolismo no Centro Hospitalar e Universitário de Coimbra (CHUC) e na Associação Protetora de Diabéticos de Portugal (APDP), com diagnóstico da doença há pelo menos um ano e com idade superior a dezoito anos. A técnica de amostragem foi por conveniência.

\section{Material}

Foram utilizados instrumentos gerais e específicos para a avaliação dos fatores comportamentais.

\section{Instrumentos gerais}

1) A escala de autoestima de Ronsenberg (RSE) (Ronsenberg, 1965). A RSE, adaptada para Portugal por Santos e Maia (2003), é um instrumento que tem como principal objetivo avaliar a autoestima de forma breve, global e unidimensional. A autoestima é entendida como autoaceitação, como sensação básica de mérito pessoal, de respeito por si próprio, como uma atitude positiva ou negativa relativamente ao self. É aplicável à população de qualquer idade. Este instrumento é constituído por dez itens, apresentado por afirmações em que as respostas são dadas numa escala ordinal de concordância com quatro posições, entre o concordo fortemente a discordo 
fortemente. A pontuação varia entre 10 e 40. Quanto maior a pontuação maior a autoestima. A consistência interna da escala é de $\alpha=0,86$.

2) WHOQOL-Bref (Canavarro, Simões, Vaz-Serra, Pereira, Rijo, Quartilho e Carona, 2006). A WHOQOL-Bref é uma versão revista e reduzida do WHOQOL - 100 (OMS, 1995) constituído por vinte e seis perguntas, das quais duas avaliam a qualidade de vida geral e as restantes vinte e quatro de forma específica, organizadas em 4 domínios. Enquanto no instrumento original cada faceta é avaliada por quatro questões, nesta versão abreviada as facetas são avaliadas apenas por uma questão. Os domínios que avalia são quatro: físico; psicológico; relações sociais e meio ambiente. A pontuação final é avaliada numa escala de 0 a 100. Quanto maior a pontuação maior a qualidade de vida em cada dimensão.A consistência interna da escala total avaliada pelo $\alpha$ de Cronbach, é de $\alpha=0,92$ e para cada dimensão $\alpha=0,87, \alpha=0,84, \alpha=0,64$ e $\alpha=0,78$, respetivamente.

3) Escala de Satisfação com o Suporte Social (ESSS) (Pais-Ribeiro, 1999). A ESSS tem como objetivo avaliar a satisfação com o suporte social percebido em adultos. A versão final é constituída por quinze frases que se apresentam como um conjunto de afirmações que estão distribuídas por quatro dimensões. Os respondentes devem assinalar o grau em que concordam ou não com as afirmações numa escala ordinal de cinco pontos. Quanto mais elevado o valor obtido, maior a satisfação com o suporte social. A pontuação varia de 15 a 75. A consistência interna dos fatores, avaliada através do a de Cronbach é a seguinte: satisfação com os amigos (mede a satisfação com as amizades) $(\alpha=0,83)$; intimidade (avalia a perceção da existência de suporte social íntimo $)(\alpha=0,74)$; satisfação com a família (mede a satisfação com o suporte social familiar existente) $(\alpha=0,74)$; atividades sociais (avalia as atividades sociais que realiza) $(\alpha=0,64)$. A consistência interna da escala total é de $\alpha=0,85$.

4) Medida de Adesão aos Tratamentos (MAT) (Delgado e Lima, 2001). A MAT é uma escala que pretende avaliar o comportamento de adesão ao tratamento. A escala é constituída por sete itens, cujas respostas são dadas numa escala ordinal de seis posições. Quanto maior a pontuação, maior a adesão.A pontuação varia de 7 a 42 . A consistência interna da escala é de $\alpha=0,74$.

5) Attitudes Towards Doctors and Medicine (EAMM) (Marteau, 1990). A EAMM ou, na versão portuguesa, Escala de Atitudes Face aos Médicos e à Medicina (EAMM) foi adaptada por Pereira e Silva (1999). O principal objetivo deste instrumento é avaliar as atitudes dos indivíduos ou grupos face aos médicos e à medicina, em utentes dos serviços de saúde. Este instrumento é constituído por dezanove itens que se distribuem por quatro subescalas: atitudes positivas face aos médicos; atitudes negativas face aos médicos; atitudes positivas face à medicina; atitudes negativas face à medicina. Cada item constitui uma afirmação em que a resposta é dada numa escala ordinal de intensidade de seis pontos que varia entre o "discordo totalmente" e o "concordo totalmente". A pontuação mais baixa corresponde a uma atitude negativa face aos médicos e medicina, e a pontuação mais elevada corresponde a uma atitude positiva face aos médicos e medicina. A consistência interna é de $\alpha=0,74$.

6) Inventário de Depressão de Beck (BDI) (Beck, Ward, Mendelson, Mock e Erbaugh, 1961) versão portuguesa de Vaz-Serra e Pio-Abreu (1973a). O BDI é um instrumento de autorresposta constituído por vinte e um grupos de afirmações que têm por objetivo medir a sintomatologia depressiva, encontrando-se esta organizada em seis tipos de sintomas: afetivos, cognitivos, motivacionais, delirantes, físicos e funcionais (padrão de sono, apetite, peso e líbido). Cada um destes grupos de afirmações é composto por quatro, cinco ou seis frases ordenadas em função da gravidade crescente do sintoma com que se relacionam. As afirmações presentes em cada conjunto dizem respeito a quatro graus de severidade inexistente, leve, moderado e grave. Estes graus permitem avaliar o nível de depressão que o indivíduo evidencia. Para tal, o sujeito deverá assinalar apenas uma afirmação em cada grupo, correspondendo essa à que considera como a melhor para descrever o modo como se sente no momento atual. Em termos psicométricos, o BDI revelou possuir boas propriedades no que toca à sua fidedignidade (Beck e Beasmesderfer, 1974 in Pinto Gouveia, 1990) e validade (Bumberry et al., in Pinto Gouveia, 1990). No presente estudo utilizou-se a versão portuguesa do BDI (Vaz-Serra e Pio Pio-Abreu, 1973a; VazSerra e Pio Pio-Abreu, 1973b), que apresenta um ponto de corte de doze, encontrando-se a população deprimida acima deste valor. Por sua vez, Kendall, Hollon, Beck, Hammem e Ingram (1987) indicam os seguintes valores de corte: 0 a 9 - normal; 10 a 20 - depressão leve (pontuações entre 10 e 17 correspondem a estados disfóricos, e superiores a 17 a estados depressivos); 20 a 30 - depressão moderada; valores superiores a 30 depressão grave. A pontuação varia entre o e 63. A consistência interna é de $\alpha=0,81$.

\section{Instrumentos específicos}

7) O Perfil de Saúde da Diabetes (DHP) (Meadows, Steen, MCColl, Eccles, Shiels, Hewison e Hutchinson, 1996). O DHP é muito utilizado em doentes com DM tipo 1 e DM tipo 2 e permite avaliar o estado de saúde do indivíduo. Contém trinta e dois itens e engloba três subescalas: perturbação psicológica (e.g. humor disfórico, hostilidade e irritabilidade); barreiras para a atividade (e.g. visível debilitação em atividades sociais); refeição desinibida (e.g. refeição em resposta ao despertar emocional), que 
medem a relação da diabetes com disfunções comportamentais e psicológicas. Este instrumento é de fácil e rápida administração e é o único instrumento que possui versões específicas para os dois tipos de diabetes. A pontuação varia numa escala de o a 100. Quanto maior o total da escala, maior a disfunção. A confiança destas subescalas foi medida pelo alfa de Cronbach em 0,86, 0,82 e 0,77 , respetivamente.

8) Questionário de Autocuidados da Diabetes (QACD) (Silva, Pais-Ribeiro, Cardoso e Ramos, 2002). O QACD avalia a adesão ao autocuidado em doentes com diabetes e é constituído por onze itens inspirados no The Summary of Diabetes Self-care Activities Questionnaire de Toolbert e Glascow (1994, cit. por, Pais-Ribeiro, 2007). Trata-se de um questionário de autorresposta que pretende avaliar a adesão ao tratamento da diabetes ao longo da última semana. Os itens estão distribuídos por três subescalas: cuidados alimentares; tratamento insulínico; tratamento antidiabético oral. As respostas são tipo Likert, com possibilidade de cinco a seis opções de resposta, conforme a subescala que está a ser avaliada. Quanto mais elevado for o resultado total das subescalas maior a adesão ao tratamento. Os valores das subescalas variam entre o e 28 (cuidados alimentares) e o e 16 (tratamento insulínico e antidiabético oral). A consistência interna do total da escala é aceitável, atendendo ao reduzido número de itens $(\alpha=0,66)$. Cada uma das subescalas tem uma consistência interna de $\alpha=0,62, \alpha=0,75, \alpha=0,88$, respetivamente.

\section{Procedimentos}

Foi solicitado aos doentes que participassem num estudo sobre a diabetes através do preenchimento de instrumentos de autorresposta.

Todos os participantes tiveram informação prévia sobre como deveriam proceder para o preenchimento dos instrumentos e, apesar de serem todos de autorresposta, sempre que solicitado por parte dos doentes estava um psicólogo ou um enfermeiro disponível para apoiar no seu correto preenchimento.

\section{Análise Estatística}

A análise estatística dos dados foi efetuada por recurso ao Software estatístico SPSS Statistics (v.21; IBM SPSS Statistics).

$\mathrm{Na}$ generalidade são apresentadas as estatísticas descritivas e inferenciais. A análise fatorial exploratória (AFE) foi realizada por recurso ao método das componentes principais. A adequação dos dados à AFE foi verificada por meio dos testes de Kaiser-Meyer-Olkin (KMO) e esfericidade de Bartlett. Note-se que o modelo testado engloba três fatores (internos, relacionais e externos ao paciente), no entanto, procedemos a uma
AFE forçada a dois fatores dado que 0 fator correspondente aos aspetos externos ao paciente não integrou a AFE pelo facto das variáveis nele incluídas serem tipo de diabetes (DM tipo 1 e DM tipo 2) e regime terapêutico (insulínico e oral).

\section{Considerações Ético-Legais}

O estudo aqui reportado foi submetido à Comissão de Ética para a Saúde dos Hospitais da Universidade de Coimbra (atualmente designado por CHUC) e Direção da Associação Portuguesa de Diabéticos de Portugal tendo sido obtido parecer positivo. Foi concedida autorização de administração de todos os instrumentos por parte dos editores e/ou autores. Todos os participantes deram o seu consentimento informado, por escrito, sendo-lhes assegurado o anonimato e confidencialidade dos dados.

\section{RESULTADOS}

Na Tabela 1 são apresentados os dados relativos ao sexo e tipologia da diabetes dos participantes no estudo.

Tabela 1

Distribuição da Amostra por Sexos e Tipo de Diabetes

\begin{tabular}{clcccc}
\hline & & \multicolumn{3}{c}{ Tipo 1 } & \multicolumn{2}{c}{ Tipo 2 } \\
\cline { 3 - 6 } Sexo & & $n$ & $\%$ & $n$ & $\%$ \\
\cline { 3 - 6 } & Masculino & 83 & 52,2 & 113 & 60,4 \\
& Feminino & 76 & 47,8 & 75 & 39,6 \\
& Total & 159 & 100 & 188 & 100 \\
\hline
\end{tabular}

Notas: $\mathrm{n}=$ amostra; \% = frequência relativa em percentagem.

\section{Estrutura fatorial exploratória}

Realizou-se uma análise fatorial exploratória com base em todos totais das escalas e subescalas por forma a tentar compreender se as variáveis compósitas dos vários instrumentos se reduziam a fatores comportamentais. Foi igualmente avaliada a adequação dos dados à análise fatorial.

O valor de KMO foi de 0,73 (DM tipo 1) e 0,70 (DM tipo 2) e o teste de Esfericidade de Bartlett alcançou em ambos os casos significância estatística, suportando a fatoribilidade da matriz de correlação. Assim, através do método da análise das componentes principais, foi possível encontrar seis a sete variáveis hipotéticas (fatores). Fatores esses que, teoricamente podiam ser, em nosso entender, determinantes para a adesão e que se enquadravam no modelo teórico a explicar.

A estrutura fatorial encontrada, através da rotação varimax e com valores próprios superiores a um, foi clara quanto ao tipo de fatores (intrínsecos e externos ao 
paciente), bem como em função do tipo de diabetes e em função do tratamento.

Apresentamos seguidamente os resultados da AFE por tipologia de diabetes e tratamento.
Na Tabela 2 são reportados os dados da AFE na DM1 tendo emergido seis fatores com valores próprios superiores a um, explicando $32,40 \%, 13,82 \%, 9,28 \%, 6,20 \%$, $5,65 \%$ e $4,65 \%$ da variância respetivamente, perfazendo um total de variância explicada de $71,96 \%$.

\section{Tabela 2}

DM1 - Análise Exploratória das Componentes Principais

\begin{tabular}{|c|c|c|c|c|c|c|}
\hline \multirow[b]{2}{*}{ Escalas e subescalas } & \multicolumn{6}{|c|}{ Componentes } \\
\hline & 1 & 2 & 3 & 4 & 5 & 6 \\
\hline Depressão & $-0,676$ & & & & & \\
\hline Autoestima & 0,670 & & & & & \\
\hline Suporte social (ESSS) & 0,385 & 0,884 & & & & \\
\hline Satisfação com os amigos (ESSS) & 0,341 & 0,819 & & & & \\
\hline Intimidade (ESSS) & 0,375 & 0,462 & & & & \\
\hline Satisfação com a família (ESSS) & & 0,727 & & & & \\
\hline Atividades sociais (ESSS) & & 0,668 & & & & \\
\hline Atitudes face aos médicos e à medicina (ADMS) & & & 0,771 & 0,569 & & \\
\hline Atitudes positivas face aos médicos & & & & 0,941 & & \\
\hline Atitudes negativas face aos médicos & & & 0,944 & & & \\
\hline Atitudes positivas face à medicina & & & & 0,941 & & \\
\hline Atitudes negativas face à medicina & & & 0,944 & & & \\
\hline Medida de adesão aos tratamentos & & & & & & 0,734 \\
\hline Perfil de saúde do diabético (DHP) & $-0,369$ & & & & 0,864 & \\
\hline Perturbação psicológica (DHP) & $-0,497$ & & & & 0,618 & \\
\hline Barreiras físicas (DHP) & & & & & 0,832 & \\
\hline Refeição desinibida (DHP) & & & & & 0,442 & 0,556 \\
\hline Autocuidado alimentar & & & & & & 0,577 \\
\hline Autocuidado tratamento insulínico & & & & & & 0,721 \\
\hline Qualidade de vida geral (WG) & 0,774 & & & & & \\
\hline Qualidade de vida - Físico & 0,733 & & & & & \\
\hline Qualidade de vida - Relações sociais & 0,751 & & & & & \\
\hline Qualidade de vida - Psicológico & 0,644 & 0,414 & & & & \\
\hline Qualidade de vida - Meio ambiente & 0,750 & & & & & \\
\hline
\end{tabular}

Notas: Rotação Varimax com normalização de Kaiser. Componentes: 1 = Psicológico; 2 = Social; 3 = Atitudes negativas; 4 = Atitudes positivas; 5 = Perfil de Saúde; 6 = Autocuidado.

Nas Tabelas 3 e 4 são apresentados os dados relativos à análise das componentes principais no caso da DM tipo 2. A Tabela 3 refere-se aos resultados em situação de tratamento insulínico, da qual resultaram sete componentes com valores próprios superiores a um, explicando 31,34\%, 11,90\%, 11,09\%, $6,77 \%, 5,55 \%, 4,56 \%$ e $4,24 \%$ da variância, respetivamente. O total da variância explicada para estes fatores/componentes situou-se nos $75,45 \%$.
Os resultados da análise das componentes principais, no caso da DM tipo 2, mas com tratamento oral são apresentados na Tabela 4. Neste último caso resultaram seis componentes com valores próprios superiores a um explicando 32,31\%, 12,90\%, 9,67\%, 8,10\%, 7,10\% e 4,94\% da variância respetivamente. A variância total explicada é de $75,03 \%$. 
Tabela 3

DM2 (insulina) - Análise das Componentes Principais

\begin{tabular}{|c|c|c|c|c|c|c|c|}
\hline \multirow[b]{2}{*}{ Escalas e subescalas } & \multicolumn{7}{|c|}{ Componentes } \\
\hline & 1 & 2 & 3 & 4 & 5 & 6 & 7 \\
\hline Depressão & $-0,558$ & $-0,435$ & & & & & \\
\hline Autoestima & 0,674 & & & & & & \\
\hline Suporte social (ESSS) & & 0,791 & & & 0,414 & & \\
\hline Satisfação com os amigos (ESSS) & & 0,810 & & & & & \\
\hline Intimidade (ESSS) & & & & & & & $-0,747$ \\
\hline Satisfação com a família (ESSS) & & 0,841 & & & & & \\
\hline Atividades sociais (ESSS) & & & & & 0,630 & & \\
\hline Atitudes face aos médicos e à medicina (ADMS) & & & 0,720 & 0,594 & & & \\
\hline Atitudes positivas face aos médicos & & & & 0,941 & & & \\
\hline Atitudes negativas face aos médicos & & & 0,960 & & & & \\
\hline Atitudes positivas face à medicina & & & & 0,941 & & & \\
\hline Atitudes negativas face à medicina & & & 0,960 & & & & \\
\hline Medida de adesão aos tratamentos & & 0,521 & & & & & 0,523 \\
\hline Perfil de saúde do diabético (DHP) & $-0,808$ & $-0,352$ & & & & & \\
\hline Perturbação psicológica (DHP) & $-0,586$ & $-0,478$ & & & & & \\
\hline Barreiras físicas (DHP) & $-0,808$ & & & & & & \\
\hline Refeição desinibida (DHP) & & & & & 0,407 & $-0,448$ & \\
\hline Autocuidado alimentar & & & & & & 0,768 & \\
\hline Autocuidado tratamento insulínico & & & & & & 0,720 & \\
\hline Qualidade de vida geral (WG) & & 0,360 & & & 0,616 & & \\
\hline Qualidade de vida - Físico & 0,534 & & & & 0,579 & & \\
\hline Qualidade de vida - Relações sociais & 0,696 & & & & 0,337 & & \\
\hline Qualidade de vida - Psicológico & & & & & 0,673 & & \\
\hline Qualidade de vida - Meio ambiente & 0,503 & & & & 0,556 & & \\
\hline
\end{tabular}

Notas: Rotação Varimax com normalização de Kaiser. Componentes: 1 = Psicológico; 2 = Social; 3 = Atitudes negativas; 4 = Atitudes positivas; 5 = Perfil de Saúde; 6 = Autocuidado; 7 = Apoio.

Tabela 4

DM2 (oral) - Análise das Componentes Principais

\begin{tabular}{|c|c|c|c|c|c|c|}
\hline \multirow[b]{2}{*}{ Escalas e Subescalas } & \multicolumn{6}{|c|}{ Componentes } \\
\hline & 1 & 2 & 3 & 4 & 5 & 6 \\
\hline Depressão & $-0,665$ & & & & & $-0,354$ \\
\hline Autoestima & 0,665 & & & & & \\
\hline Suporte social (ESSS) & & & & 0,768 & & 0,543 \\
\hline Satisfação com os amigos (ESSS) & & & & 0,838 & & \\
\hline Intimidade (ESSS) & & & & & & 0,769 \\
\hline Satisfação com a família (ESSS) & & & & 0,906 & & \\
\hline Atividades sociais (ESSS) & & & & & & 0,835 \\
\hline Atitudes face aos médicos e à medicina (ADMS) & & 0,737 & 0,624 & & & \\
\hline Atitudes positivas face aos médicos & & & 0,951 & & & \\
\hline Atitudes negativas face aos médicos & & 0,906 & & & & \\
\hline Atitudes positivas face à medicina & & & 0,951 & & & \\
\hline Atitudes negativas face à medicina & & 0,906 & & & & \\
\hline Medida de adesão aos tratamentos & & & & & 0,839 & \\
\hline Perfil de saúde do diabético (DHP) & $-0,913$ & & & & & \\
\hline Perturbação psicológica (DHP) & $-0,798$ & & & & & \\
\hline Barreiras físicas (DHP) & $-0,748$ & & & & 0,331 & \\
\hline Refeição desinibida (DHP) & $-0,609$ & & & & & \\
\hline Autocuidado alimentar & & & & & 0,763 & \\
\hline Autocuidado tratamento oral & & & & & 0,759 & \\
\hline Qualidade de vida geral (WG) & 0,715 & & & & & ,305 \\
\hline Qualidade de vida - Físico & 0,674 & & & 0,305 & & \\
\hline Qualidade de vida - Relações sociais & 0,771 & & & & & \\
\hline Qualidade de vida - Psicológico & 0,713 & & & 0,324 & & 0,390 \\
\hline Qualidade de vida - Meio ambiente & 0,766 & & & & & \\
\hline
\end{tabular}

Notas: Rotação Varimax com normalização de Kaiser. Componentes: 1 = Psicológico; 2 = Social; 3 = Atitudes negativas; 4 = Atitudes positivas; 5 = Perfil de Saúde; 6 = Apoio. 


\section{Análise das componentes principais (ACP) forçada a}

dois fatores. Como se apresenta na Tabela 5 explorou-se a forma como se agrupavam os construtos latentes deste estudo. Assim, procedeu-se a uma ACP forçada a dois fatores, de forma testar o modelo teórico composto por três grandes fatores: internos, relacionais e externos ao paciente. Para valores próprios superiores a um, os dois fatores explicam $32,36 \% 13,82 \%$ da variância. A variância total explicada é de $46,17 \%$.

Tabela 5

DM 1 - ACP Forçada a Dois Fatores

\begin{tabular}{|c|c|c|}
\hline & \multicolumn{2}{|c|}{ Componente } \\
\hline & 1. Interno & 2. Relacional \\
\hline Depressão & $-0,808$ & \\
\hline Autoestima & 0,696 & \\
\hline Suporte Social & 0,847 & \\
\hline Suporte Social - Satisfação com amigos & 0,735 & \\
\hline Suporte Social - Intimidade & 0,658 & \\
\hline Suporte Social - Família & 0,559 & \\
\hline Suporte Social - Atividades sociais & 0,660 & \\
\hline Atitudes face aos médicos e à medicina & & 0,930 \\
\hline Atitudes positivas face aos médicos & & 0,674 \\
\hline Atitudes negativas face aos médicos & & 0,703 \\
\hline Atitudes positivas face à medicina & & 0,674 \\
\hline Atitudes negativas face à medicina & & 0,703 \\
\hline Medidas de adesão ao tratamento & 0,345 & \\
\hline Perfil de saúde do diabético & $-0,767$ & \\
\hline Perfil de saúde - perturbação psicológica & $-0,757$ & \\
\hline Perfil de saúde - barreiras físicas & $-0,474$ & \\
\hline Perfil de saúde - refeição desinibida & $-0,537$ & \\
\hline Autocuidado alimentar & 0,331 & 0,361 \\
\hline Autocuidado tratamento insulínico & & 0,408 \\
\hline Qualidade de vida geral & 0,688 & \\
\hline Qualidade de vida - domínio físico & 0,639 & \\
\hline Qualidade de vida - domínio psicológico & 0,723 & \\
\hline Qualidade de vida - domínio das relações sociais & 0,783 & \\
\hline Qualidade de vida - domínio do meio ambiente & 0,698 & \\
\hline Variância explicada & $32,359 \%$ & $13,815 \%$ \\
\hline
\end{tabular}

Total de variância explicada $46,174 \%$

Nota: Rotação Varimax com normalização de Kaiser

De acordo com o modelo estabelecido, o "autocuidado alimentar" e o "tratamento insulínico" deveriam saturar na componente interna, contudo, como se observa na Tabela 6 , tal não se verifica.

Na Tabela 7 pode verificar-se que a satisfação com o suporte social satura de forma bem manifesta na componente "interna" e não na "relacional".

A variância explicada situa-se nos 31,34\%, 11,90\% e $43,24 \%$ da variância total, no caso da DM tipo 2 com tratamento oral. No que respeita ao tratamento insulínico a variância é de 32,31\%, 12,90\% e 45,21\% da variância total.
Tabela 6

DM 2 (insulina) - ACP Forçada a Dois Fatores

\begin{tabular}{|c|c|c|}
\hline & \multicolumn{2}{|c|}{ Componente } \\
\hline & 1. Interno & 2. Relacional \\
\hline Depressão & $-0,777$ & \\
\hline Autoestima & 0,684 & \\
\hline Suporte Social & 0,779 & 0,334 \\
\hline Suporte Social - Satisfação com amigos & 0,635 & 0,476 \\
\hline Suporte Social - Intimidade & 0,402 & \\
\hline Suporte Social - Família & 0,532 & 0,347 \\
\hline Suporte Social - Atividades sociais & 0,498 & \\
\hline Atitudes face aos médicos e à medicina & 0,466 & \\
\hline Atitudes positivas face aos médicos & & 0,615 \\
\hline Atitudes negativas face aos médicos & 0,385 & $-0,680$ \\
\hline Atitudes positivas face à medicina & & 0,615 \\
\hline Atitudes negativas face à medicina & 0,385 & $-0,680$ \\
\hline Medidas de adesão ao tratamento & 0,493 & \\
\hline Perfil de saúde do diabético & $-0,819$ & \\
\hline Perfil de saúde - perturbação psicológica & $-0,814$ & \\
\hline Perfil de saúde - barreiras físicas & $-0,604$ & \\
\hline \multicolumn{3}{|l|}{ Perfil de saúde - refeição desinibida } \\
\hline \multicolumn{3}{|l|}{ Autocuidado alimentar } \\
\hline \multicolumn{3}{|l|}{ Autocuidado tratamento insulínico } \\
\hline Qualidade de vida geral & 0,701 & \\
\hline Qualidade de vida - domínio físico & 0,672 & \\
\hline Qualidade de vida - domínio psicológico & 0,769 & \\
\hline Qualidade de vida - domínio das relações sociais & 0,594 & \\
\hline Qualidade de vida - domínio do meio ambiente & 0,819 & \\
\hline Variância explicada & $31,343 \%$ & $11,897 \%$ \\
\hline Total de variância explicada & \multicolumn{2}{|c|}{$43,240 \%$} \\
\hline
\end{tabular}

Tabela 7

DM 2 (oral) - ACP Forçada a Dois Fatores

\begin{tabular}{|c|c|c|}
\hline & \multicolumn{2}{|c|}{ Componente } \\
\hline & 1.Interno & 2Relacional \\
\hline Depressão & $-0,776$ & \\
\hline Autoestima & 0,612 & \\
\hline Suporte Social & 0,758 & \\
\hline Suporte Social - Satisfação com amigos & 0,650 & \\
\hline Suporte Social - Intimidade & 0,514 & \\
\hline Suporte Social - Família & 0,475 & \\
\hline Suporte Social - Atividades sociais & 0,493 & \\
\hline Atitudes face aos médicos e à medicina & & 0,951 \\
\hline Atitudes positivas face aos médicos & & 0,603 \\
\hline Atitudes negativas face aos médicos & & 0,709 \\
\hline Atitudes positivas face à medicina & & 0,603 \\
\hline Atitudes negativas face à medicina & & 0,709 \\
\hline Medidas de adesão ao tratamento & & 0,336 \\
\hline Perfil de saúde do diabético & $-0,768$ & \\
\hline Perfil de saúde - perturbação psicológica & $-0,756$ & $-0,355$ \\
\hline Perfil de saúde - barreiras físicas & $-0,624$ & \\
\hline Perfil de saúde - refeição desinibida & $-0,389$ & $-0,391$ \\
\hline Autocuidado alimentar & 0,301 & \\
\hline Autocuidado tratamento oral & & 0,304 \\
\hline Qualidade de vida geral & 0,768 & \\
\hline Qualidade de vida - domínio físico & 0,696 & \\
\hline Qualidade de vida - domínio psicológico & 0,719 & \\
\hline Qualidade de vida - domínio das relações sociais & 0,870 & \\
\hline Qualidade de vida - domínio do meio ambiente & 0,751 & \\
\hline Variância explicada & $32,312 \%$ & $12,899 \%$ \\
\hline Total de variância explicada & & $211 \%$ \\
\hline
\end{tabular}


De ressalvar que esta estrutura denota boas qualidades psicométricas dos instrumentos utilizados. A análise forçada a dois fatores evidenciar que os itens saturam, na sua maioria, de forma mais carregada nos fatores a que teoricamente pertencem, exceção feita para a subescala intimidade que deveria ter saturado na dimensão relacional.

\section{DISCUSSÃO E CONCLUSÕES}

Os resultados que emergiram da análise exploratória dos totais das escalas e subescalas demonstraram a existência de várias dimensões/fatores comportamentais. Assim, no caso da DM tipo 1, a estrutura encontrada foi de seis fatores denominados psicológico; social; atitudes negativas; atitudes positivas; perfil de saúde e autocuidado. No que respeita ao fator psicológico os construtos que lhe correspondem são a depressão, autoestima e qualidade de vida; ao fator social corresponde-lhe apenas o suporte social; ao fator atitudes negativas são as atitudes face aos médicos e medicina (escala total), atitudes negativas face aos médicos e à medicina; fator atitudes positivas são as atitudes positivas face aos médicos e à medicina; fator perfil de saúde é o perfil de saúde do diabético excetuando a subescala relativa à refeição desinibida; fator autocuidado engloba as medidas de adesão ao tratamento, refeição desinibida, autocuidado alimentar e tratamento insulínico. Assim, os resultados obtidos nesta primeira análise exploratória apontam para a inclusão de aspetos relacionados com a sintomatologia depressiva, o construto avaliativo da autoestima enquanto orientação positiva ou negativa do sujeito em referência a si mesmo (Rosenberg, 1965). De acrescentar a dimensão da qualidade de vida englobando esta a saúde física, o estado psicológico, o nível de independência, as relações sociais, as crenças pessoais e as suas relações com aspetos do meio envolvente (WHOQOL Group, 1995). Já no que se refere ao fator social, o suporte social remete para a ideia de existência ou disponibilidade de outros que se preocupam, confiam, e nos valorizam (Pais-Ribeiro, 1999). Os demais fatores encontrados correspondem ao que seria expectável face aos instrumentos utilizados, sendo de salientar que o fator autocuidado integra fundamentalmente os apetos inerentes à alimentação e tratamento sendo estes indicadores indiretos de adesão.

No que respeita à $\mathrm{DM}$ tipo 2 com tratamento insulínico, a estrutura fatorial aumenta para sete fatores denominados psicológico; social; atitudes negativas; atitudes positivas; qualidade de vida; autocuidado e, por último, apoio. De notar que as variáveis latentes saturam nas componentes adequadas, exceção feita para o suporte social - intimidade e medidas de adesão ao tratamento que saturam na componente apoio. Resulta desta análise exploratória a omissão da componente perfil de saúde, cujas variáveis latentes saturam no fator psicológico e autocuidado. A refeição desinibida, comportamento alimentar e terapêutico encontram-se isoladas do fator que denominámos de autocuidado. Outro dado interessante diz respeito à qualidade de vida relações sociais que não satura de forma mais carregada na componente qualidade de vida, mas sim na componente psicológica. Uma possível explicação poderá ser a de que as relações sociais avaliadas por esta subescala têm os itens redigidos com base numa avaliação da satisfação pessoal e opinativa, assente na ideia de como o indivíduo se relaciona com os outros. Por exemplo, destacamos os itens estou satisfeito com a quantidade de tempo que passo com os meus amigos, não saio tantas vezes quantas eu gostaria. Esta avaliação por parte de quem responde está assente numa autoperceção sobre si na relação com os outros, pelo que é natural que se agregue à componente psicológica. No que concerne à intimidade, as questões são geralmente baseadas numa avaliação de apoio, e não propriamente de proximidade emocional, caso do item 1 por vezes sinto-me só no mundo e sem apoio, item 4 quando preciso de desabafar com alguém encontro facilmente amigos com quem o fazer, item 5 mesmo nas situações mais embaraçosas, se precisar de apoio de emergência tenho várias pessoas a quem posso recorrer, item 6 às vezes sinto falta de alguém verdadeiramente íntimo que me compreenda e com quem possa desabafar sobre as coisas íntimas.

Reduz-se a seis fatores a AFE no caso da DM tipo 2 terapêutica oral. Assim, considerou-se as seguintes componentes psicológica; atitudes negativas; atitudes positivas; social; autocuidado e apoio. É inequívoca a saturação das variáveis latentes depressão, perfil de saúde e qualidade de vida na primeira componente, isto é, psicológico, bem como as atitudes negativas e atitudes positivas nas componentes 2 e 3 respetivamente. Mais uma vez, a intimidade se isola do fator a que teoricamente deveria pertencer, já que é uma dimensão da ESSS. Contudo, tal como referido anteriormente, a justificação pode estar associada à forma como os itens estão redigidos. Neste caso, na DM tipo 2 com tratamento oral, a subescala atividades sociais associa-se à componente 6 , tal como a intimidade. A explicação mantém-se baseada na forma como o doente se sente apoiado pelos amigos, exemplo do item 14 estou satisfeito com as atividade e coisas que faço com o meu grupo de amigos.

A denominação das componentes que resultaram da AFE foi assumida de forma independente, uma vez que se considerou adequado, do ponto de vista metodológico, adotar uma estrutura fatorial com as mesmas denominações quando todo o estudo assenta numa análise diferenciada em função do tipo de DM e terapêutica. 
$\mathrm{Na}$ tentativa de perceber como se agrupavam as variáveis latentes (escalas e subescalas) foi efetuada uma ACP forçada a dois fatores. Desta feita foi examinada a existência do fator interno e relacional. Conclui-se que o modelo a dois fatores está devidamente encontrado. Com efeito, o construto apoio social não satura no fator relacional, mas sim no fator interno. A ESSS é uma escala manifestamente utilizada em investigações, e cujas propriedades psicométricas se revelam de grande qualidade, contudo, atendendo à forma como os itens estão redigidos, o doente tende a responder de forma muito mais pessoal/individual do que relacional. Sabe-se que as relações sociais são em tudo complexas, que é difícil a destrinça entre o eu e os outros, entre o que são reais necessidades, o que é o suporte real sentido e o suporte social recebido.

Cramer, Henderson e Scott (1997) distinguem suporte social percebido do suporte social recebido. O primeiro refere-se ao suporte social que o indivíduo percebe como disponível se precisar dele, enquanto o segundo descreve o suporte social recebido por alguém.

Uma outra distinção aceitável é o que se entende por suporte social descrito e suporte social avaliado. O suporte social descrito refere-se à presença de um tipo específico de comportamento de suporte, já o segundo, assenta numa base avaliativa desse mesmo comportamento de suporte que pode ser satisfatório e, por isso mesmo, sentido como uma ajuda. Depreende-se facilmente, que a importância individual que atribuímos a tão vasto construto fundamente os resultados encontrados, sobretudo no que se refere à intimidade e relações com os amigos.

No que concerne a estudos no âmbito da saúde, destacamos a investigação levada a cabo por Wethingston e Kessler (1986) que pretenderam determinar se as variáveis de saúde estavam associadas com a perceção de suporte social, propriamente dito, ou com os aspetos objetivos do social. Verificaram que os resultados de saúde eram melhor explicados pela perceção de suporte social do que pelos aspetos tangíveis. Deste modo, e de acordo com Kessler (1985, cit. por Pais-Ribeiro 1999) no caso das doenças crónicas, o suporte social refere-se aos mecanismos pelos quais as relações interpessoais conseguem proteger os indivíduos dos efeitos prejudiciais do stresse sendo que esta variável é muito abrangente, englobando um vasto conjunto de componentes e de aspetos revestindo-se de procedimentos de avaliação muito diversos. Considera-se que esta possível diversidade de avaliação tenha contribuído para uma limitação ao estudo. Acresce a esta reflexão as eventuais limitações do recurso a instrumentos de autorresposta que assentam em pressupostos teóricos complexos e com respostas previamente categorizadas.
A literatura reporta que a adesão ao tratamento na doença crónica implica cumprir escrupulosamente as recomendações terapêuticas (WHO, 2003; Nieuwlaat, et al., 2008). A adesão além de ter um efeito positivo na manutenção da saúde, pressupõe aceitação da doença (Sgnaolin e Figueiredo, 2012). Por ser um conceito dinâmico, complexo e multidimensional (Rosner, 2006; Fine, Becker, De Geest, et al. 2009) deverá ser entendido tendo por base componentes internas, relacionais e externas ao paciente, tal como explicitado neste artigo. Estas evidências poderão ser um contributo para delinear estratégias educacionais e comportamentais de promoção à adesão.

Conflito de interesses | Conflict of interest: nenhum | none. Fontes de financiamento | Funding sources: nenhuma | none.

\section{REFERÊNCIAS}

Beck, A. Ward, C. Mendelson, M., Mock, J. e Erbaugh, J. (1961). An inventory for measuring depression. Archives of General Psychiatry, 4, 561-571.

Brooks, L. M. (2002). Perceived barriers to treatment adherence among pregnant African American women with diabetes. Doutoramento, Case Western Reserve University, Health Sciences Cleveland, Ohio.

Canavarro, M. C., Simões, M. R., Vaz-Serra, A., Pereira, M., Rijo, D., Quartilho, M. J. e Carona, C. (2007). Instrumento de avaliação da qualidade de vida da Organização Mundial de Saúde: WHOQOL-Bref. In M. Simões, C. Machado, M. Gonçalves e L. Almeida (Eds.), Avaliação psicológica: Instrumentos validados para a população portuguesa (Vol. III, pp. 77-100). Coimbra: Quarteto Editora.

Cramer, D., Henderson, S. e Scott, R. (1997). Mental health and desired social support: a four-wave panel study. Journal of Social and Personal Relationships, 14(6), 761-775.

Delgado, A. B. e Lima. M. L. (2001). Contributo para validação concorrente de uma medida de adesão aos tratamentos. In Psicologia, Saúde e Doenças, 2(2), 81-100.

Fine, R.N., Becker, Y., De Geest S., Einsen, H., Ettenger, R, Evans, R. ... Dobbels, F. (2008). Nonadherence Consensus Conference Summary Report. American Journal of Transplantation, 9(1), 35-41.

Hirschberg, S. L. (2001). The self-regulation of health behavior in children with insulin-dependent diabetes mellitus. Doutoramento, Faculty of Pacific Graduate School of Psychology, Palo Alto, California.

Kendall, P. C., Hollon, S. D., Beck, A. T., Hammem, C. L. e Ingram, R. E. (1987). Issues and recommendations regarding the use of the Beck Depression Inventory. Cognitive Therapy and Research, 11, 289-299.

Leventhal, H. e Cameron, L. (1987). Behavioral theories and the problem of compliance. Patient Education and Counseling, 10, 117-138. doi: 10.1016/0738-3991(87)90093-0.

Marteau, T. M. (1990). Attitudes to doctors and medicine: The preliminary development of a new scale. Psychology and Health, 4, 351-356.

Meadows, K. A., Abrams, C. e Sandbaek, A. (2000). Adaptation of diabetes health profile (DHP-1) for use with patients with type $2 \mathrm{DM}$ : psychometric evaluation and cross-cultural comparison. Diabetic Medicine, 17, 572-580.

Meadows, K. A., Steen, N., MCColl E., Eccles. M., Shiels C., Hewison J. e Hutchinson, A. (1996). The Diabetes Health Profile (DHP): a new instrument for assessing the psychosocial profile of insulin requiring 
patients-development and psychometric evaluation. Quality of Life Research, 5, 242-254.

Nieuwlaat, R., Wilczynski, N., Navarro, T., Hobson, N., Jeffery, R., Keepanasseril, A., ... Haynes, R. B. (2008). Interventions for enhancing medication adherence. In R. Nieuwlaat (Ed.), Cochrane Database of Systematic Reviews. Chichester, UK: John Wiley e Sons, Ltd. doi:10.1002/14651858.CDooo011.pub3

Pais-Ribeiro, J. L. (1999). Escala de satisfação com o suporte social (ESSS). Análise psicológica, 3(XVII), 547-558.

Pais-Ribeiro, J. L. (2007). Avaliação em psicologia da saúde: instrumentos publicados em português. Porto: Livpsic.

Pasquali, L. (2003). Psicometria: Teoria dos Testes na Psicologia e na Educação. Petrópolis: Vozes.

Pereira, M. G. e Silva, N. S. (1999). Escala de atitudes face aos médicos e à medicina. In A. Soares, S. Araújo e S. Caires (Org.s). Avaliação psicológica: formas e contextos (pp.496-503). Braga: APPORT.

Pinto-Gouveia, J. (1990). Factores Cognitivos de Vulnerabilidade para a Depressão (Estudo da sua interacção com os acontecimentos de vida). Doutoramento, Universidade de Coimbra, Coimbra.

Rosenberg, M. (1965). Society and the adolescent self-image. Princeton: Princeton University Press.

Rosner, F. (2006). Patient noncompliance: causes and solutions. The Mount Sinai Journal of Medicine, New York, 73(2),553-559.

Ryan, C. M. (1997). Psychological factors and diabetes mellitus. In J. Pickup e G. Williams (Ed.), Textbook of diabetes (pp. 66.1-66.13). Oxford: Blackwell Science.

Sackett, D. L. e Haynes, R. B. (1976). Compliance with therapeutic regimens. Baltimore, MD, Johns Hopkins University Press.
Sanchez-Sosa, J. J. (2001). Treatment adherence: the role of behavioral mechanisms operating through health care interventions. Paper presented in the meeting. Geneva: World Health Organization. Adherence to Long-Term Therapies: Toward Policies for Action

Santos, P. J. e Maia, J. (2003). Análise factorial confirmatória e validação preliminar de uma versão portuguesa da escala de auto-estima de Rosenberg. Psicologia: Teoria, Investigação e Prática, 2, 253-268.

Sgnaolin, V. e Figueiredo, A. (2012). Adesão ao tratamento farmacológico de pacientes em hemodiálise. Jornal Brasileiro de Nefrologia, 34(2), 109-116. doi:10.1590/S0101-28002012000200002

Silva, I., Pais-Ribeiro, J., Cardoso, H. e Ramos, H. (2002). Questionário de autocuidados na diabetes - contributo para a criação de um instrumento de avaliação da adesão ao tratamento. Psiquiatria clínica, 23(3), 227-237.

Vaz-Serra, A. e Pio-Abreu, J.L. (1973a). Aferição dos quadros clínicos depressivos. I - Ensaio de aplicação do "inventário depressivo de Beck" a uma amostra portuguesa de doentes deprimidos. Coimbra Médica, XX, 623-644.

Vaz-Serra, A. e Pio Pio-Abreu, J.L. (1973b). Aferição dos quadros clínicos depressivos. II - Estudo preliminar de novos agrupamentos sintomatológicos para complemento do "inventário depressivo de Beck" a Coimbra Médica, XX, 713-736.

Warren, L. e Hixenbaugh, P. (1998). Adherence and diabetes. In MYERS, L. B.; MIDENCE, K., ed. lit., Adherence to treatment in medical conditions. Netherlands: Harwood Academic Publishers. p. 423-453.

Wethingston, E. e Kessler, R. C. (1986). Perceived support, received support, and adjustment to stressfull life evets. Journal of Health and Social Behavior, 27, 78-89.

WHO (2003). Adherence to long-term therapies. Evidence for action. WHO. (Cap. XIII, pp. 107-114). 